\title{
Morfologia externa de Thyridia psidii cetoides (Rosenberg \& Talbot). I. Cabeça e apêndices (Lepidoptera, Nymphalidae, Ithomiinae) '
}

\author{
Jorge Manuel Saraiva Bizarro ${ }^{2}$, Mirna Martins Casagrande ${ }^{2} \&$ Olaf Herman Hendrik Mielke ${ }^{2}$ \\ 1 Contribuição número 1361 do Departamento de Zoologia, Universidade Federal do Paraná. \\ 2 Departamento de Zoologia, Universidade Federal do Paraná. Caixa Postal 19020, 81531-980 Curitiba, Paraná, Brasil. \\ Bolsista CNPq. E-mail: bizarro@xmail.com.br, mibras@ufpr.br, omhesp@ufpr.br
}

\begin{abstract}
External morphology of Thyridia psidii cetoides (Rosenberg \& Talbot). l. Cabeça e apêndices (Lepidoptera, Nymphalidae, Ithomiinae). A detailed study of the morphology of the head of Thyridia psidii cetoides (Rosenberg \& Talbot, 1914) (Nymphalidae, Ithomiinae) adults from both sexes is presented. The material was obtained at the city's plant nursery “Horto Florestal de Curitiba”, Paraná, Brazil; mainly by rearing eggs and larvae collected there on Cyphomandra betacea (Canavilles) Sendtner, 1845 (Solanaceae). When possible, all the results obtained were compared with those already available in the literature concerning external morphology studies pertinent to other Nymphalidae subfamilies (Brassolinae, Morphinae and Danainae).
\end{abstract}

KEY WORDS. Head, Ithomiinae, morphology, Neotropical, Nymphalidae, Thyridia.

A classificação de Lepidoptera para as categorias taxonômicas superiores, especialmente família, evoluiu consideravelmente desde a criação do sistema de nomenclatura zoológica por LinNaEus (1758). A controvérsia atual entre os especialistas na ordem versa sobre a atribuição de categorias taxonômicas superiores aos diversos grupos naturais já definidos.

As propostas mais recentes para a sistemática de Lepidoptera são as de HeppNer (1996) e Kristensen (1999), sendo que o primeiro não hesita em afirmar que no momento atual, qualquer tentativa de estabelecer uma classificação adequada se reveste forçosamente de um caráter provisório. A causa principal é o conhecimento pouco detalhado e sistematizado, solidamente fundamentado, da morfologia comparada de imaturos e adultos, ontogenia e ecologia; aliado por sua vez ao escasso conhecimento das propriedades e escopo de variabilidade dos caracteres da taxonomia molecular. Esse panorama é duplamente agravado pela grande diversidade morfológica e pelo colossal número de espécies em algumas das famílias na ordem, dificultando aos lepidopteristas o estabelecimento de caracteres substanciais, sobre os quais se possa construir uma classificação mais estável.

Entre as borboletas diurnas, a família mais numerosa é Nymphalidae, que apesar de estabelecida taxonomicamente como um grupo natural, nos níveis inferiores à categoria família não está isenta de controvérsia sistemática, estando ainda longe de uma classificação satisfatória. Existem muitos grupos naturais bem definidos, mas as relações entre eles continuam obscuras, em parte porque na sistemática de Lepidoptera se tem, desde há muito, tendência a usar um grupo particular de caracteres, ignorando, por questão de metodologia ou tradição, outros eventualmente pertinentes. Assim, assiste-se ao aparecimento de muitas tentativas de classificação baseadas em combinações de caracteres diferentes, sendo os resultados díspares, conforme o conjunto usado. Conclusivamente, EHRLICH (1958b), influenciado na época pela escola fenética, defendia a necessidade de proceder a um estudo básico e completo da morfologia de representantes dos diversos grupos naturais, usando o maior número possível de caracteres na resolução dos problemas taxonômicos.

Dentro dessa linha e na seqüência de outros trabalhos relativos à morfologia externa de membros da família Nymphalidae, o objetivo do presente é proceder ao estudo da morfologia externa de um membro basal da subfamília Ithomiinae, tribo Mechanitini (Fox 1949, 1967, D' AlmEIDA 1978, MielKe \& K. BRown 1979): Thyridia psidii cetoides (Rosenberg \& Talbot, 1914).

Estudos morfológicos detalhados para espécies das seguintes subfamílias de Nymphalidae foram utilizados para comparação: Danainae (Ehrlich 1958a): Danaus plexippus plexippus (Linnaeus, 1758), Brassolinae (CASAGRANDE 1979a, b, c, d): Caligo beltrao (Illiger, 1801), Morphinae (BıьоттA 1993, 1995a, b): Morpho helenor violaceus Fruhstorfer, 1912; Iphimedeia hercules (Dalman, 1823); Iphixibia anaxibia (Esper, [1801]); Cytheritis portis thamyris (C. Felder \& R. Felder, 1867); Cytheritis aega (Hübner, [1822]); Pessonia catenaria (Perry, 1811); Grasseia menelaus nestira (Hübner, 1821)

EhrLich (1958b) efetuou um estudo de aproximadamente 600 espécies da superfamília Papilionoidea, que inclui a família Nymphalidae, visando uma tentativa de classificação filoge- 
nética geral da mesma com base na morfologia externa. É, sem dúvida, um trabalho pioneiro, mas os resultados foram publicados de forma muito sumária, sem figuração/iconografia abrangente da morfologia pesquisada, talvez por sua finalidade não ser uma morfologia comparada, mas uma tentativa de estabelecimento das relações filogenéticas dentro de Papilionoidea.

\section{MATERIAL E MÉTODOS}

\section{Origem do material estudado}

Os adultos foram obtidos de duas criações em cativeiro para garantir a integridade morfológica do material a analisar. A primeira criação (piloto) foi levada a cabo durante os meses de abril a junho de 1996 (outono), mediante a obtenção de imaturos de Thyridia psidii cetoides (ovos e larvas) coletados sobre a planta hospedeira - Cyphomandra betacea (Canavilles) Sendtner, 1845, tomate de árvore, no Horto Florestal de Curitiba, Paraná; acondicionados posteriormente em caixas de plástico com fundo coberto por papel absorvente e folhas frescas da planta hospedeira, à temperatura ambiente. As caixas foram inspecionadas regularmente, sendo limpas e fornecidas folhas novas quando necessário. A segunda criação foi realizada durante o mês de janeiro de 1997 (verão) em casa de vegetação, sobre mudas de Cyphomandra betacea confinadas em vasos, provenientes do Horto Florestal, nas condições de umidade e temperatura ambientais. Providencialmente, essas mudas vieram com ovos de Thyridia psidii cetoides, tendo sido o restante do material coletado em visitas regulares a um único pé da planta, existente no Horto Florestal da cidade.

\section{Preservação e estudo do material}

Adultos proveniente das criações foram mortos em frasco letal e fixados em Dietrich, de forma a não danificar as estruturas do exoesqueleto para o estudo morfológico; parte dele foi depositado na coleção de Entomologia Padre Jesus Santiago Moure, Departamento de Zoologia, Setor de Ciências Biológicas, Universidade Federal do Paraná (DZUP) (Figs 1-4).

Procedeu-se à dissecação do material, retirando-o do líquido de Dietrich, e fervendo-o numa solução de 10\% de hidróxido de potássio $(\mathrm{KOH})$, em banho-maria, até obtenção do seu amolecimento. As escamas foram removidas com a ajuda de pincel e estiletes. As peças foram separadas nos respectivos tagmas - cabeça, tórax e abdome - deixando intacto o segmento visado.

O estudo do material foi realizado em placa de Petri com parafina escavada de forma a ser possível a cobertura de toda a peça pela água.

\section{Confecção dos desenhos}

Os desenhos foram efetuados com a ajuda de estereomicroscópio acoplado à câmara clara. Para as estruturas da cabeça foi usado um microscópio no exame e desenho de peças mais delicadas.

Salvo indicação oposta, a escala de cada desenho representa $1 \mathrm{~mm}$.

\section{Nomenclatura utilizada}

Para a morfologia dos adultos seguiu-se o padrão descritivo e comparativo dos trabalhos já publicados para a família Nymphalidae: Danainae (Ehrlich 1958a, b; baseada em Duporte 1956 e Matsuda 1965), Brassolinae (CASAgrande 1979a, b, c, d) e
Morphinae (Bı́отт 1993, 1995a, b), com particular ênfase para as diferenças encontradas, a fim de permitir uma maior facilidade em estudos posteriores. Para a venação das asas usouse ainda o trabalho de Nielsen \& Common (1991).

\section{RESULTADOS E DISCUSSÃO}

\section{Cabeça (Figs 5-11)}

A cabeça apresenta um par de olhos compostos, hemisféricos, sendo a sua largura superior à altura.

Área central frontal ocupada pelo frontoclípeo de forma semi-circular, superiormente limitado pela sutura transfrontal. Em Thyridia psidii cetoides esta sutura é bem marcada externamente e curta, ocupando a região entre os soquetes antenais à semelhança de Danaus plexippus (ЕнrцICH 1958a), notoriamente diferente de Caligo beltrao (CASAgRande 1979b) e de várias espécies de Morphinae (Bilotta 1993), onde se posiciona anteriormente à inserção das antenas; na região dorsal do soquete antenal, uma saliência - o antenífero - serve de ponto de articulação para o escapo. Lateralmente, o frontoclípeo é limitado pela sutura ocular (CASAGRANDE 1979b) - ou sutura láterofacial sensu EHRLICH (1958a) - em forma de curva sigmóide, esta contendo as fóveas tentoriais anteriores desde a margem dorsolateral do labro à região ventral dos soquetes antenais.

O bordo ventral do clípeo apresenta uma banda esclerotinizada, $a$ faixa transclipeal (EHrLIcH 1958a), a qual tem cerca de um quinto da espessura da de Danaus plexippus (EHrLich 1958a), estando aparentemente ausente em Caligo beltrao (CASAgrande 1979b) e várias espécies de Morpho (BilotTa 1993). O limite inferior do clípeo é constituído pela sutura clipeolabral, que o separa do labro.

Regiões entre a sutura ocular e a margem dos olhos, são conhecidas como áreas paraoculares (MiCHENER 1952). Estas áreas são largas em Danaus plexippus (EHrLICH 1958a) e Thyridia psidii cetoides, mas quase ausentes em Caligo beltrao (CASAgrande 1979b) e várias espécies de Morphinae (BilotтA 1993). No limite inferior da área paraocular pode-se observar, atrás e lateralmente ao pilífero, um pequeno tubérculo, o rudimento mandibular.

A face ventral, entre os olhos, é ocupada pela fosseta proboscidial, uma região escavada, não muita profunda em Thyridia psidii cetoides.

Na face posterior, uma cavidade, o forame occipital, é bissectado pela ponte pós-occipital, originando dois forames. O superior, com um terço da altura do inferior, tem forma trapezoidal. Este forame é relativamente maior em Thyridia psidii cetoides e Danaus plexippus (Енrцich 1958a) do que em Caligo beltrao (CASAgRande 1979b) e as espécies de Morphinae (BilotTa 1993). Nestes últimos, a tendência é a de apresentar o forame superior de forma triangular ou oval e o inferior triangular ou retangular. Nas extremidades do bordo superior desta ponte encontram-se os côndilos occipitais, duas estruturas proeminentes em forma de aletas semicirculares onde se articulam os escleritos cervicais e, no seu bordo inferior, as fóveas posteriores do tentório. Ao longo do bordo superior, o forame é marginado pelo pós-occipício, estrutura mediana bem delimitada pela sutura pós-occipital, que emerge lateralmente do occipício, esclerito que ocupa todo o espaço entre o forame e as margens oculares. O bordo inferior do forame é constituído pelo prolongamento posterior do lábio, do qual se separa mediante a sutura paralabial (ЕнRцich 1958a). 

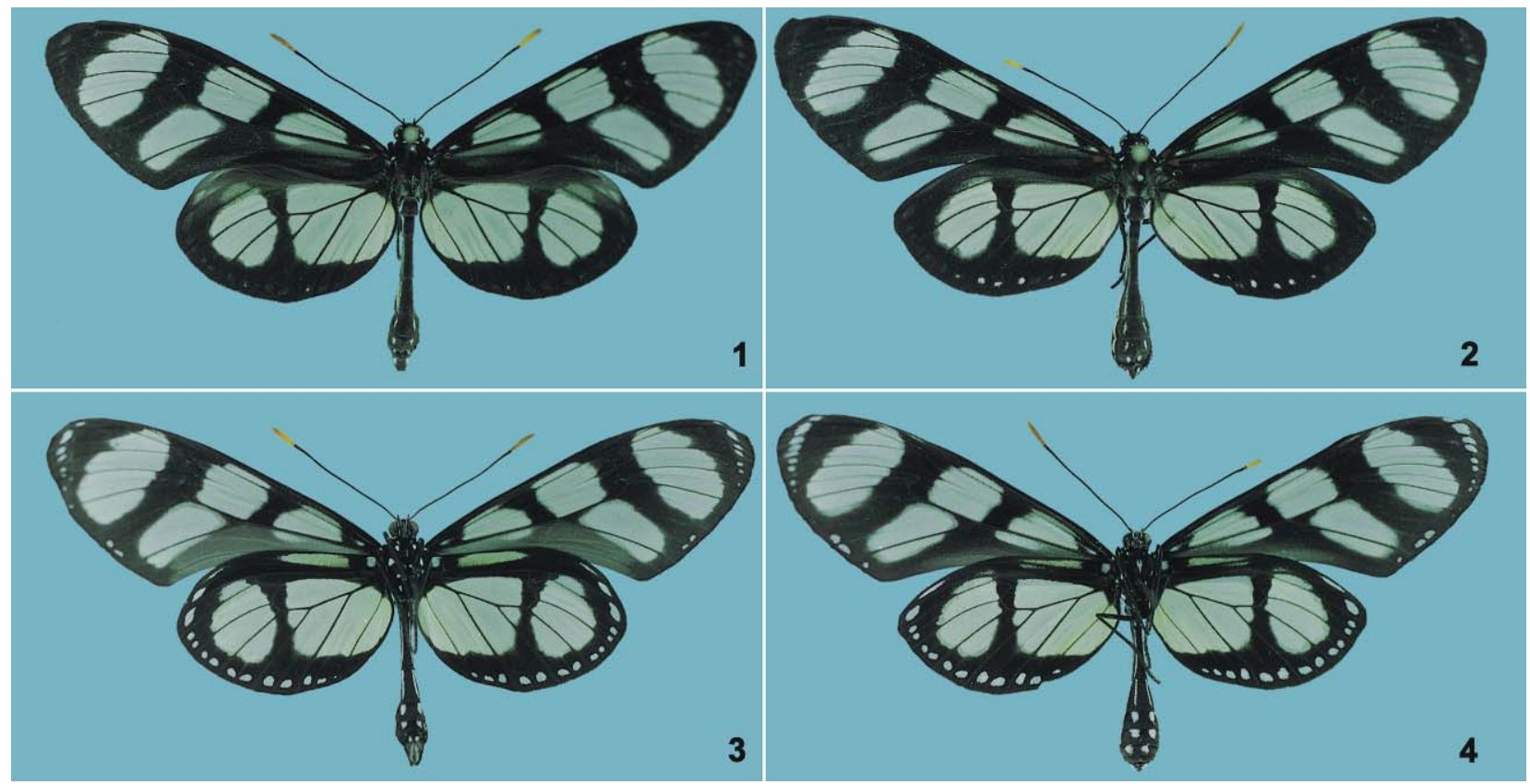

Figuras 1-4. Thyridia psidii cetoides. (1-2) Macho: (1) vista dorsal, (2) vista ventral; (3-4) fêmea: (3) vista dorsal, (4) vista ventral.

O vértice, na região dorsal da cabeça, é delimitado anteriormente pela sutura transfrontal e soquetes antenais, láterodistalmente pela sutura ocular (temporal sensu auct.). Láteroposteriormente ao escapo, entre a sutura ocular e a região lateral do vértice, tem origem uma pequena área reniforme dilatada proximalmente, de superfície espiculada, o quetosema. Este encontra-se aparentemente ausente em Danaus plexippus (Ehrlich 1958a), Caligo beltrao (Casagrande 1979b) e espécies de Morphinae (BilotTa 1993).

\section{Apêndices Cefálicos}

\section{Antenas (Figs 5, 7 e 9)}

As antenas, situadas nas margens dorso-laterais do esclerito frontoclipeal, são longas, clavadas e típicas de Papilionoidea (EHrLICH 1958b), não apresentando dimorfismo sexual.

São constituídas por um artículo basal, o escapo, em forma de anel largo, que se articula com o pedicelo, ou segundo artículo da antena, com cerca de um terço do tamanho do escapo. O restante da antena é composto pelo flagelo, que apresenta um número variável de flagelômeros, 37 ou 39 quer no macho quer na fêmea, sendo que, em muitos exemplares, o primeiro e segundo artículos individualizam-se exclusivamente nas suas faces dorsais, ocorrendo fusão dos mesmos ventralmente. Quando esta condição ocorre, o primeiro tem cerca de duas vezes o comprimento dos seguintes. Do nono artículo em diante, aumentam progressivamente, até ficarem semelhantes ao primeiro. A partir do $27^{\circ}$, a sua largura aumenta e diminui o comprimento, formando a região clavada da antena. Nestes últimos artículos, aparecem três carenas ventrais distintas, típicas da família Nymphalidae (ЕнrLICH 1958b). No entanto, em Thyridia psidii cetoides elas surgem apicalmente, somente nos 11 últimos artículos, por comparação com Danaus plexippus (Ehrlich 1958a), Caligo beltrao (CASAgrande 1979b) e espécies de Morphinae (BiLotTa 1993).

\section{Labro (Fig. 5)}

Esclerito ímpar, mediano, estreito e transversal, fortemente esclerotinizado e articulado com o frontoclípeo através da sutura clipeo-labral. Apresenta um par de estruturas cerdosas triangulares nas extremidades laterais, os pilíferos e, ventralmente ao centro, a epifaringe, pequeno lobo apical triangular pouco esclerotinizado.

\section{Maxilas (Fig. 8)}

Compostas por um par de gáleas proeminentes, alongadas e côncavas no plano sagital, de modo a formar um canal por justaposição de ambas - a espiritromba, ocupa a região anterior da fosseta proboscidial (EhrLich 1958a). Na base das mesmas encontram-se os estipes, cada um deles com um pequeno tubérculo, logo atrás e lateralmente às gáleas. Na região basal, o cardo, pequeno esclerito de forma triangular.

\section{Lábio (Figs 7, 8, 10 e 11)}

Entre o par de estipes, na região mediana posterior da fossa proboscidial, encontra-se um esclerito triangular de base posterior, o lábio, apresentando distalmente os soquetes de inserção dos palpos labiais. Na linha mediana deste esclerito observa-se uma pequena invaginação, o apódema labial, que nesta espécie tem a forma de uma cunha com base posterior. Lateralmente ao lábio e às maxilas, separando estas estruturas do olho, encontra-se a área hipostomal.

Os palpos labiais (Figs 10 e 11) são triarticulados, com forma cilíndrico-cônica, de maior diâmetro na base. O primeiro 

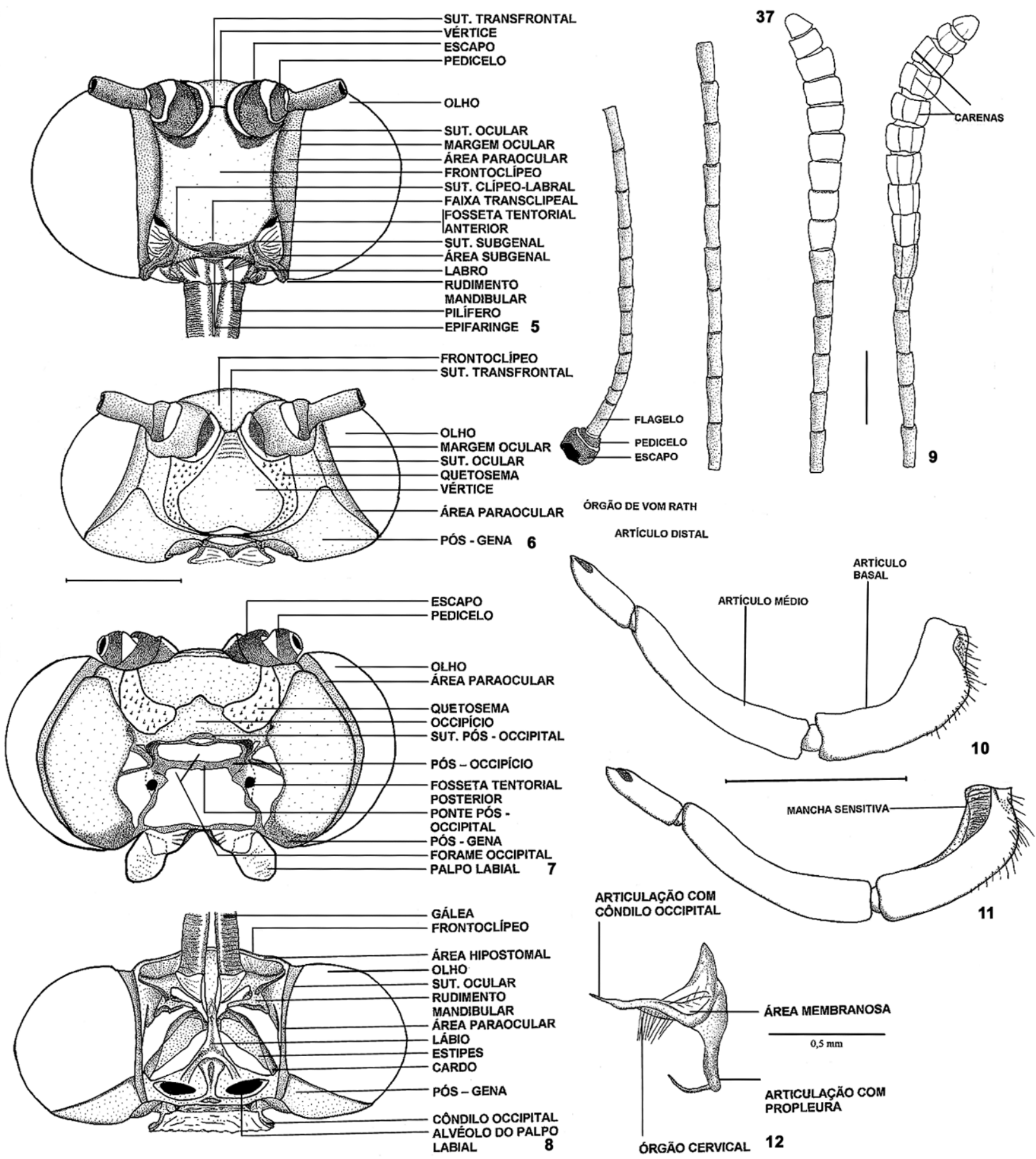

Figuras 5-12. Thyridia psidii cetoides. (5-8) Cabeça: (5) vista anterior, (6) vista dorsal, (7) vista posterior, (8) vista ventral; (9) antenas; (10-11) palpos labiais: (10) vista externa, (11) vista interna; (12) esclerito cervical, vista lateral.

Revista Brasileira de Zoologia 20 (2): 279-284, junho 2003 
artículo, mais robusto, articulando-se com o respectivo soquete, tem pouco mais de $3 / 4$ do comprimento do segundo; apresentando uma área triangular crenulada de vértice inferior na face interna, a área sensitiva, e a superfície com rugosidades no terço basal com numerosas cerdas no bordo póstero-inferior, cujo tamanho diminui progressivamente em direção anterior. O segundo artículo, maior que os restantes, com curvatura menos pronunciada, termina em bisel de superfície lisa. Terceiro artículo, o menor, com um terço do comprimento do anterior, afilado, com uma pequena área ovalada distal escura em seu interior, o órgão de vom Rath.

Nas espécies de Morphinae (BıLотta 1993), o palpo labial é de perfil quase retilíneo, sendo o primeiro artículo muito curto, com menos de um terço do comprimento do segundo. Em Danaus plexippus (EHRLICH 1958a) e Caligo beltrao (CASAGRANDE 1979b), o perfil é curvo, semelhante ao de Thyridia psidii cetoides, mas o primeiro artículo também é curto, à semelhança do que ocorre com as espécies de Morphinae.

\section{Região cervical (Fig. 12)}

Segundo Matsuda $(1965,1970)$, a região cervical teria uma origem embriológica mista, formando-se mediante a fusão de um elemento cefálico occipital com outro proveniente do protórax.

Apresenta-se totalmente membranosa, excetuando a presença de um par de escleritos cervicais em forma de ' $\mathrm{T}$ ' deitado. A extremidade anterior articula-se com a região mais externa da ponte pós-ocipital e o seu bordo posterior, a parte superior do ' $\mathrm{T}$ ', com a propleura. O esclerito é constituído por uma haste vertical projetando no terço superior dois prolongamentos anteriores que se unem por uma membrana fracamente esclerotinizada, com uma dilatação membranosa no centro do bordo inferior, na qual se inserem as cerdas do órgão cervical, sendo a extremidade anterior articulada com o côndilo occipital; apresenta ainda uma projeção inferior curta, delgada e dirigida anteriormente em direção mediana, fazendo um ângulo agudo com o prolongamento superior do esclerito.

Os escleritos cervicais, à semelhança do que ocorre em Danaus plexippus (Ehrlich 1958a), Caligo beltrao (CASAGRANDE 1979c) e nas espécies de Morphinae (BıLotTa 1995a), não se fundem na região anterior; no entanto, estes não apresentam a projeção inferior.

\section{AGRADECIMENTOS}

À Agência de Cooperação Espanhola, pela Bolsa de Mestrado concedida ao abrigo do Programa MUTIS de Cooperação Ibero-Americana. À coordenação do Curso de Pósgraduação em Ciências Biológicas - Entomologia, da Universidade Federal do Paraná, pelo apoio concedido ao longo do Curso de Mestrado, na pessoa do então coordenador Prof. Dr. Cláudio J.B. de Carvalho; e Engenheira Érika Costa Mielke do Horto Florestal de Curitiba, pela ajuda prestada na obtenção das plantas hospedeiras e coleta dos imaturos nas instalações do referido Horto.

\section{REFERÊNCIAS BIBLIOGRÁFICAS}

ACKeRY; P.R.; R. DE Jong \& R.I. VANe-Wright 1999. The butterflies: Hedyloidea, Hesperioidea and Papilionoidea, p. 263-300. In: M. FisHer (Ed.). Handbook of Zoology 4, Lep. 1. Berlin, Walter de Gruyter, 491p.
BiLotTA, I.G. 1993. Morfologia comparada da cabeça das espécies sulbrasileiras de Morphinae (Lepidoptera, Nymphalidae). Revista Brasileira de Zoologia, Curitiba, 9 (3/4): 261-271.

. 1995a. Morfologia comparada do tórax das espécies sulbrasileiras de Morphinae (Lepidoptera, Nymphalidae). Revista Brasileira de Zoologia, Curitiba, 11 (4): 691-713.

- 1995b. Morfologia comparada do abdome das espécies sulbrasileiras de Morphinae (Lepidoptera, Nymphalidae). Revista Brasileira de Zoologia, Curitiba, 11 (4): 737748.

Casagrande, M.M. 1979a. Sobre Caligo beltrao (Illiger). I: Taxonomia, biologia, morfologia das fases imaturas e distribuições espacial e temporal. (Lepidoptera, Satyridae, Brassolinae). Revista Brasileira de Biologia, Rio de Janeiro, 39 (1): 173-193.

. 1979b. Sobre Caligo beltrao (Illiger). II: Morfologia externa da cabeça do adulto. (Lepidoptera, Satyridae, Brassolinae). Revista Brasileira de Biologia, Rio de Janeiro, 39 (1): 223-227.

- 1979c. Sobre Caligo beltrao (Illiger). III: Morfologia externa do adulto - tórax. (Lepidoptera, Satyridae, Brassolinae). Revista Brasileira de Biologia, Rio de Janeiro, 39 (2): 347-355.

. 1979d. Sobre Caligo beltrao (Illiger). IV: Morfologia externa do adulto - abdome. (Lepidoptera, Satyridae, Brassolinae). Revista Brasileira de Biologia, Rio de Janeiro, 39 (2): 347-355.

D'AlmeIDA, R.F. 1978. Catálogo dos Ithomiidae Americanos (Lepidoptera). Curitiba, Centro de Recursos Audiovisuais, Universidade Federal do Paraná, IV+405p.

DuPorte, E.M. 1956. The median facial sclerite in larval and adult lepidoptera. Proceedings of the Royal Entomological Society of London (A) 31: 109-116.

EHRLICH, P.R. 1958a. The integumental anatomy of the monarch butterfly Danaus plexippus L. (Lepidoptera - Danaidae). The University of Kansas Science Bulletin, Lawrence, 38 (18): 1315-1349.

.1958b. The comparative morphology, philogeny and higher classification of the butterflies. The University of Kansas Science Bulletin, Lawrence, 39: 305-370

Fox, R.M. 1949. The evolution and systematics of the Ithomiidae (Lepidoptera). University of Pittsburgh Bulletin, 45 (8): 36-47.

. 1956. A monograph of the Ithomiidae (Lepidoptera). Part I. Bulletin of the American Museum of Natural History, New York, 111: 1-76.

1967. A monograph of the Ithomiidae (Lepidoptera). Part III, the tribe Mechanitini, Fox. Memoirs of the American Entomological Society, Philadelphia, 22: 1-190.

Heppner, J.B. 1993 [1996]. Keys to families of Lepidoptera. Tropical Lepidoptera, Gainesville, 4 (Suppl. 3): 1-28.

Kristensen, N.P. 1999. (ver P. Ackery; de Jong \& R.I. Vane Wright, 1999)

LINNAEUS, C. 1758. Systema naturae per regna tria naturae, secundum classes, ordines, genera, species cum characteribus, differentiis, synonymis, locis. Regnum Animale I. Holmiae, Laurentii Salvii, Editio decima, reformata, 824p.

Revista Brasileira de Zoologia 20 (2): 279-284, junho 2003 
MatsudA, R. 1965. Morphology and Evolution of the Insect Head. Memoirs of the American Entomological Institute, Gainesville, 4 (8): 1-334.

. 1970. Morphology and Evolution of the Insect Thorax. Memoirs of the Entomological Society of Canada, Ottawa, 76: 334-355.

Michener, C.D. 1952. The Saturniidae (Lepidoptera) of the Western Hemisphere, morphology, phylogeny, and classification. Bulletin of the American Museum of Natural
History, New York, 98 (5): 335-502.

Mielke, H.H. \& K.S. Brown JR. 1979. Suplemento ao Catálogo dos Ithomiidae Americanos (Lepidoptera) de Romualdo Ferreira d'Almeida (Nymphalidae: Ithomiinae). Curitiba, Centro de Recursos Audiovisuais, Universidade Federal do Paraná, VII+216p.

Nielsen, E.S. \& I.F.B. Common. 1991. Lepidoptera, p. 765-866. In: CSIRO (Ed.). The Insects of Australia. Reed Carlton, Melbourne University Press, 1137p.

Recebido em 26.IX.2002; aceito em 27.V.2003.

Revista Brasileira de Zoologia 20 (2): 279-284, junho 2003 